\title{
Corrigendum: Megafaunal isotopes reveal role of increased moisture on rangeland
} during late Pleistocene extinctions

M. Timothy Rabanus-Wallace, Matthew J. Wooller, Grant D. Zazula, Elen Shute, A. Hope Jahren, Pavel Kosintsev, James A. Burns, James Breen, Bastien Llamas and Alan Cooper

Nature Ecology \& Evolution 1, 0125 (2017); published 18 April 2017; corrected 5 June 2017.

The Supplementary Information files that were originally published with the Article were not the intended final files. Both files have been updated. 